toothed gin trap is advocated on these and other grounds.

The evidence for a nine-eleven-year cycle of rabbit numbers in Britain was strong, until myxomatosis appeared. The virtual disappearance of the rabbit may benefit the hare because the rabbit is a reservoir for diseases affecting both animals and is, of course, also a grazing competitor. However, the rabbit will not disappear completely; myxomatosis is now enzootic in Australia and France and is appearing sporadically in recovering populations in Britain, where the rabbit's own flea, spilopsyllus cuniculi, has proved the only competent vector. To the benefit of agriculture and forestry we may now expect low numbers for years ahead.

The authors consider that it is doubtful if natural predators are of importance in the control of rabbits except in special circumstances.

This is an important book, bringing as it does right up to date the latest research on both the rabbit and myxomatosis, as well as a full chapter on the legal aspects of rabbit control, as pest and game.

R. M. LOCKLEY

\section{THE STRUCTURE OF TURBULENT SHEAR FLOW}

The Structure of Turbulent Shear Flow

By Dr. A. A. Townsend. Pp. xii $+315.8 \frac{3}{4}$ in. $\times 5 \frac{1}{2}$ in. (Cambridge: At the University Press.) $40 s$.

7 URBULENCE occurs so frequently in the world around us and is so grossly chaotic in appearance that the layman is inclined to accept it simply as one of the vagaries of Nature. However, turbulence plays such a significant part, not only in Nature, but also in our technological machines, that we are led to seek statistical regularity and predictability within the chaos of turbulence. For the first third of the present century, turbulence research was dominated by concepts of mixing lengths, which, since they were formulated in terms of mean flow properties, were immediately applicable to the solution of practical problems. However, as more information became available about the dynamical behaviour of the turbulence itself, it was found that concepts of mixing lengths were not consistent with the observed behaviour. It thus became necessary to rebuild turbulence theory in conformity with this revised picture of the structure of turbulence.

Turbulence research is carried on in only a few laboratories throughout the world. One of the bestknown is the Cavendish Laboratory at Cambridge. There, over the past decade, Dr. A. A. Townsend has been engaged in a systematic investigation of turbulent flows using some of the more advanced techniques currently available in hot-wire anemometry. With Sir Geoffrey Taylor and Dr. G. K. Batchelor, Dr. Townsend has been in the forefront in providing information which undermined the earlier theories of turbulence. In an effort to build a sound theory of turbulence, Dr. Townsend has now written the monograph under notice. He has succeeded admirably in constructing a theory which appears to stand on a firm foundation.

The central framework of the book consists of the experimental observations which have been made by Dr. Townsend and others on the following turbulent flows : isotropic turbulence, homogeneous turbulence undergoing uniform deformation, wakes, jets, flows in pipes and channels, boundary layers with and without pressure gradients and flows between rotating cylinders. Behind these experimental observations, there lurk subtle concepts such as Reynolds's number similarity, self-preserving flows, moving equilibrium and the like that have formed in the minds of the men familiar with the data. Dr. Townsend has done an excellent job of formulating these concepts and showing the part they play in the interpretation of the observations and in the construction of theories consistent with the observations.

From the experimental observations, Dr. Townsend has sought out a picture of the structure of turbulent flows. Earlier, the view had emerged that for isotropic turbulence there exists a range of eddies of intermediate size which contain the major portion of the turbulent energy, and a range of eddies of small size which are responsible for the viscous conversion of turbulent energy to heat. For shear flows, Dr. Townsend adds a range of large eddies of simple structure which are responsible for the large-scale convection. Using this structural picture, he constructs an analytical description of shear turbulence which is convincingly consistent with the experimental observations of both the turbulent and the mean flow fields.

The overall result is a remarkably able discussion of the difficult problem of turbulent shear flow. This monograph will undoubtedly stand as a cornerstone for much of the serious research on shear flows in years to come.

Francis H. Clauuser

\section{CHEMISTRY OF HYDROGEN PEROXIDE}

\section{Hydrogen Peroxide}

By Prof. Walter C. Schumb, Prof. Charles N. Satterfield and Ralph L. Wentworth. (American Chemical Society Monograph Series, No. 128.) Pp. xiii +759. (New York: Reinhold Publishing Corporation; London : Chapman and Hall, Ltd., 1955.) 132s, net. CINCE its discovery in 1818 by Thenard, hydrogen $\checkmark$ peroxide has occupied a unique position in the scheme of chemistry on account of the nature of its chemical bond and its usefulness to the study of catalysis. As the knowledge of its properties increased it was realized that hydrogen peroxide was not an inherently unstable substance and the development of concentrated hydrogen peroxide in Germany during the Second World War as a military propellant demonstrated that the material could be handled on a technical scale and in large quantities. The present book, which covers all aspects of hydrogen peroxide chemistry and technology, is the most exhaustive survey of the existing knowledge on this compound which has yet been written. The authors and their co-workers have during the past decade made a considerable contribution to a wide varioty of problems concerned with hydrogen peroxide and they write from a deep and extensive knowledge of technique and experiment with this material. Although reference is made to more than 2,500 published articles the book is not solely a compilation of datathe treatment throughout is selective, thorough, critical, and no aspect of the subject is omitted.

The book begins with the early history and technical development of hydrogen peroxide. This is followed by descriptions of the present-day methods of formation and manufacture from water or oxygen 